\title{
Non-Delay Tolerant Non-Overlay Routing Protocols Performance Evaluation for VANET
}

\author{
Mahmoud Ali Al Shugran ${ }^{1}$ \\ ${ }^{1}$ Department of Computer Seines and Information Technology, Jerash University, Jordan \\ Correspondence: Mahmoud Ali Al Shugran, Department of Computer Seines and Information Technology, Jerash \\ University, Jordan.
}

Received: August 2, 2021

Accepted: August 26, 2021

Online Published: September 3, 2021

doi:10.5539/cis.v14n4p20

URL: https://doi.org/10.5539/cis.v14n4p20

\begin{abstract}
A Vehicular Ad hoc Network (VANET) is a distinctive situation of wireless ad hoc networks. The designing of the routing protocol considers a critical role in communication in VANET. VANET has specific features compared to other types of wireless ad hoc networks that impose special characteristics for designing of efficient routing protocols.The challenging factor in designing efficient routing protocols for VANET is the high movement of vehicles that incurs a rapid change in the network topology that causes frequent link breakage. This paper presents and evaluates different position-based routing protocols associated with VANETs. The evaluation aiming to determine appropriate specifications for optimal routing protocols' features achieving best performance within different environmental conditions. The performance comparison is carried out in terms of Packet Delivery Rate (PDR), Void Problem Occurrence Rate (VPOR), and Average Hops Count (AHC).
\end{abstract}

Keywords: vehicular Ad hoc network, routing protocols, network topology, link breakage, PDR, VPO, AHC

\section{Introduction}

Vehicular ad hoc networks (VANETs) (Asim, R., Saira, G., Sana, A., \& Amir, Q. 2017) are a particular category of wireless ad hoc networks. The vehicle (or nodes) in VANETs forms the keystone of this network. The communication between participating nodes in VANET is executed through two modes. The first mode is carried out between two vehicles $(\mathrm{V} 2 \mathrm{~V})$. The second mode is accomplished between vehicles Infrastructure communication (V2I) (Joilson, A. J., Emilio, C., \& Wille, G. 2018). To set up and preserve communication, vehicles exchange data between them as well as with Road Side Units (RSUs). The participating nodes are provided with wireless transceivers, to communicate with each other and with RSUs. The V2V communication can be in the same or in the opposite direction. The VANETs are self-configuring, thus to provide communications between nodes, VANET in needs to deploy Intelligent Transportation Systems (ITS). The ITS can emanate the communication with transportation for controlling the traffic, to support vehicular safety, and to mitigate traffic congestion (Debasis, D., \& Rajiv, M. 2018).

VANET applications are split into two categories: Safety based and non-safety-based applications (Asim, R., Saira, G., Sana, A., \& Amir, Q. 2017). The first category is subdivided into, vehicular traffic efficiency and infotainment applications (Joilson, A. J., Emilio, C., \&Wille, G. 2018). In addition to the safety applications, VANETs offered non-safety based such as real-time information to the road users like weather information, multimedia applications, and mobile e-commerce. For the sake of those applications efficient and continuous exchanging of information is in need. In VANET, communication can be accomplished between vehicles directly or through multi-hop patterns. The VANET has several challenges due to its unique characteristics that may degrade the communication process between nodes (Debasis, D., \& Rajiv, M. 2018).These problems have motivated researchers to suggest new and enhanced proposal that satisfies the new VANET's challenges and requirements.

The major contribution of this research concentrates on the V2V communication in VANETs. Further, unicast location-based routing protocols are the focal of this research. This research aims to investigate some well-known position-based routing protocols. The research introduces a comparison between popular selected protocols in urban and highway environments. The remainder of the paper is sort as follows: Section 2 discusses some recent related works. Section 3 is dedicated to a detailed classification of unicast position-based routing protocols. Next, section 4 reviews some selected protocols belonging to the unicast position-based routing 
protocols. Section 5 shows the performance evaluation setup. Section 6 discusses simulation comparison and discussion of the selected protocols by giving a particular interest to specific performance metrics. Section 7 concludes this study and suggests directions for the future paper to enhance the performance of communication over VANET.

\section{Related Work}

The traditional routing protocols of wireless ad hoc network aren't appropriate to be applied in VANETs due to the distinct features of VANETs. Therefore, many routing protocols have been proposed to meet VANETs requirements. Thereafter, several studies conducted to evaluate the performance of proposed routing protocols to prove their convenient to VANETs environments.

The authors of(Amardeep, D., Harmandeep, S., Sanjay, B., \&Sanjeev, S. 2013, June) made a comparison between three topology-based routing protocols. The study showed that topology-based routing protocols may suit well with VANETs with some modifications to the routing algorithm.Author (Singh, P. 2014, April)considered unicast and multicast routing and applied different data rates to compare their performance in VANET. The comparison is done by employing different routing metrics such as routing overhead, delay, and other to measure the performance of protocols under study. The research presented by author of (Manvendra, S. 2015)makes a comparison of several position-based routing protocols for VANETs. The research concentrated on several features of each routing protocol. The research concluded a qualitative comparison between selected protocols under study.

The authors of (Ali, K., Phillips, I., \& Yang, H. 2016, December) provided a comparison and evaluation of three well-known routing protocols for VANET. The evaluation was carried out for the city environment setup. Three QoS metrics were used to measure the performance of compared protocols.The experiments were carried out using two different simulators. The authors concluded that position-based routing protocols outperformed topology-based protocols. In the research presented by authors of (Zineb, S. H. \&Imane, Z. 2017), researchers explore several routing protocols proposed in the literature, including proactive, reactive, hybrid topology-based routing protocols and position-based protocols. To evaluate the performance of selected protocols different things were considered. Thus, the study was acomplished under a various number of participating vehicles and plenty of traffics load. in an urban scenario. The study concluded that position-based were more appropriate to VANETs compared to topology-based protocols.

Authors of (Smiri, S., Boushaba, A., Ben A. R., \&Zahi, A. 2018, May) researchers compared and evaluated the performance of seven ad-hoc routing protocols for VANETs. The study considered specific routing metrics and performance metrics too. The study concluded that position-based is more convenient to VANEts compared to topology-based routing protocols. Researchers of (Amina, B., Asmae, B., Mohamed, E. 2020) provided a detailed taxonomy of routing protocols in VANETs. The researchers explored and identified the pros and cons of each category. Next, researchers compared and evaluated selected routing protocols of each category. The evaluation is achieved by considering three quality of service metrics. The researchers concluded that position-based routing protocols may be more appropriate to VANETs by considering other routing metrics besides distance metric.

In spite of the understanding of researchers and the different proposals to compare evaluate existing routing protocols performance in VANET. There is a shortage of in-depth performance evaluation of proposed position-based routing protocols that take into consideration the special features of VANETs.Thus, this research work compares and evaluates the latest position-based that were proposed to be suited to different VANETs environments.

\section{Classifications of Unicast Routing Protocols in VANET}

In VANET, efficient routing protocols have to employ unique designs that guarantee reliable communication between two communicating nodes with no disruption. This section discusses the two main categories of VANET routing protocols. The first category uses global information about the network topology and the information about the communication links between participating nodes. Those two metrics are used to make forwarding decisions. Thus, participating vehicles preserve a neighbours' routing table (NRT). Vehicles consult their NRT before making a forwarding decision. The second category employs neighbouring geographical position datum to execute data packet routing (Anil, K. T. \& Ali T. R. 2016). Figure 1 shows the two well-known branches for data packet forwarding for VANETs which are topology-based and position-based routing protocols. 


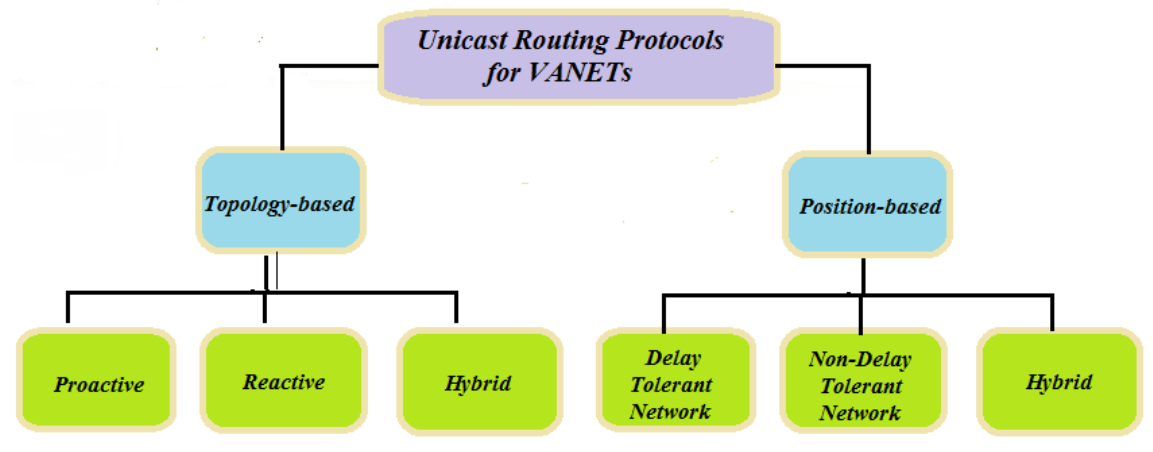

Figure 1. The well-known categories for data packet forwarding for VANETs

\subsection{Topology-based Routing Protocols}

Topology-based protocols utilize the information about links between participating nodes in VANET. The collected information is exploited to carry out the data packet routing. Therefore, all participating vehicles should maintain a neighbouring routing table (NRT). These protocols are subdivided into proactive (table-driven), reactive (on-demand), and hybrid approaches. Literature showed that traditional topology-based routing protocols have a lot of drawbacks that make them unsuitable as routing approaches in VANETs.This research focuses on available unicast position-based routing protocols (Smiri, S., Boushaba, A., Ben A. R., \&Zahi, A. 2018, May).

\subsection{Position-based Routing Protocols}

The major precondition for location-based routing is that any participating node has the ability to gain its current geographic location, and neighborhood, and the final targeted vehicle. Thus, the routing decisions require location services such as the Global Position System (GPS). The GPS is employed to locate the position of the participating nodes. Through using GPS, every vehicle can continuously send beacon packets with its position information. Compared to the topology-based approach, Position-based protocols are suitable for VANETs due to the features that make them attain a higher packet delivery rate, and showed a reduction in delay and overhead (Kaur, H., \&Meenakshi, M. 2017, May). Three procedures recognize the operation of position-based routing protocols which are; Path selection, forwarding mechanism, and recovery strategy.

Most current location-based protocols employ a greedy forwarding mechanism (GFM) to forward data packets between communicating vehicles. Nodes that use GFM need to know the geographical location of the final targeted vehicle as well as neighbourhood location datum. Source node that has data to be sent selects next-hop based on an optimized criteria of the algorithm. In case GFM fails to forward packets and the data suffers from the void problem, the protocol tunes to recovery mode. Based on the recovery methods, employed routing protocols are subdivided into three categories. Those categories are; Delay Tolerant Network (DTN), Non-Delay Tolerant Network (Non-DTN), and Hybrid (Anil, K. T. \& Ali T. R. 2016). The (GeOpps) (Leontiadis, I., \&Mascolo, C. 2007, June)Geographical Opportunistic Routing and Vehicle-Assisted Data Delivery (VADD) (Zhao, J. \& Cao, G. 2008) are well-known routing protocol belong to DTN. The NON-DTN protocols subdivided into three categories, namely, beacon, no-beacon, and hybrid protocols.

The beacon category is sub classified into two branches namely; overlay and non-overlay mechanisms (Kaur, H., \&Meenakshi, M. 2017, May). This paper presents NON-DTN and the Non-overlay beacon category. The Non-overlay method makes forwarding decisions hop-by-hop. Proposed routing protocols belong to this category tried to solve routing issues in VANET. One of the earlier routing protocols that apply the non-overlay approach is the Greedy Perimeter Stateless Routing (GPSR) (Karp, B. \& Kung, H. T. 2000, August). Furthermore, this category holds other routing protocols such as; Position-Based Routing with Distance Vector Recovery (PBR-DV) (Kirsch, B., \&Effelsberg W. 2007) that uses On-Demand Distance Vector Routing (AODV)(Perkins, C.E. \& Royer, E.M 1999, February),Reliability-Based GPSR Protocol: GPSR-R (Shelly, S., \&Babu, A.V. 2015), Greedy Perimeter Stateless Routing with Movement Awareness (GPSR-MA) (Fabrizio, G., Giulia, B., Dzmitry, K., \& Gianni V. 2007), Greedy Perimeter Stateless Routing with lifetime for VANETs (GPSR-L) (Rao, S. A., Pai, M., Boussedjra, M., \&Mouzna, J. 2008, October), A Reliability-Improving Position-Based Routing (RIPR) (Ryu, M. W. 2011), and Greedy Routing with Abstract Neighbour Table (GRANT) (Sascha, S., \& Wolfgang, E. 2008). 


\section{Representative Non-DTN Non-Overlay Routing Protocols}

This paper will discuss several unicast Non-DTN Non-Overlay position-based routing protocols, and examine their performance. Figure 2 shows the surveyed protocols.

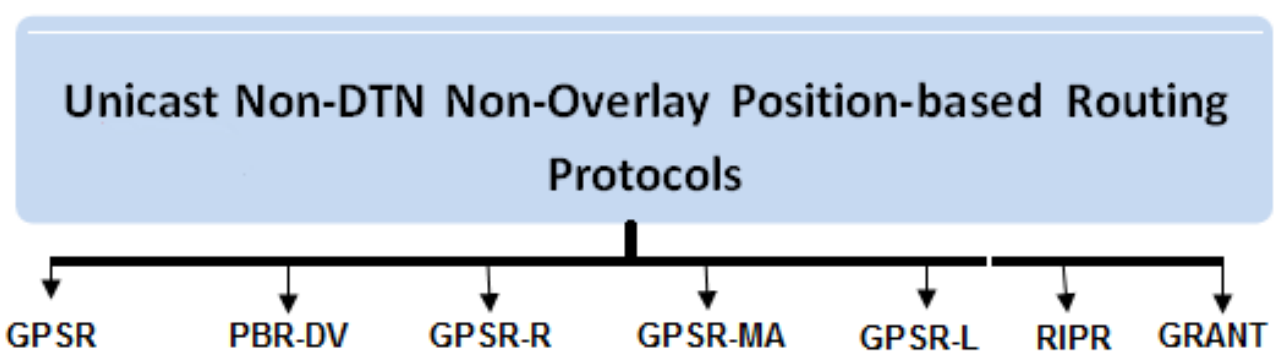

Figure 2. The Surveyed Unicast Non-DTN Non-Overlay Protocols

\subsection{Representatives Protocols in Highway Environment}

This section discusses some well-known position-based routing protocols that are deployed in highway environments.

\subsubsection{Greedy Perimeter Stateless Routing (GPSR)}

GPSR (Karp, B. \& Kung, H. T. 2000, August) represents the cornerstone of several proposed routing protocols in VANET. The GPSR algorithm, as a position-based protocol, concerns about participating nodes' location.The functionality of GPSR depends on two modes: greedy forwarding mechanism (GFM), and recovery mode. In GFM forwarded packets are routed to a neighbour vehicle that satisfies the GFM selection criterion based on the distance routing metric. The routed data follow a hop-by-hop process until it reaches its final target. If GFM fails to find the next-hop, GPSR switches to the second operation by using recovery mode by applying the right-hand rule to route around voids.

\subsubsection{Position-Based Routing with Distance Vector Recovery (PBR-DV)}

In (Kirsch, B., \& Effelsberg W. 2007) authors proposed the Position-Based Routing with Distance Vector Recovery (PBR-DV) routing protocol. The PBR-DV algorithm makes use of the greedy forwarding strategy and the On-Demand Distance Vector Routing (AODV) (Perkins, C.E. \& Royer, E.M. 1999, February) recovery approach. The superior idea of PBR-DV starts when a packet falls in void. At this point the PBR-DV protocol switches to AODV to forwards the data packet.

\subsubsection{Reliability-Based GPSR Protocol (GPSR-R)}

The authors in (Shelly, S., \& Babu, A.V. 2015) proposed a new model that adopted link status between communicating vehicles. The design is called reliability-based GPSR (GPSR-R). Hello Messages (HeM) were transmitted regularly within fixed intervals of time. The HeM holds information that belongs to the sender vehicle such as current velocity (speed and motion direction), in addition to current geographical location. A vehicle that receives HeM builds its own neighbours' routing table (NRT). A vehicle that needs to send data consults its NRT to find out the most reliable link with its current neighbours. Evaluation result for the GPSR-R protocol ensures that it outperforms conventional GPSR in terms of link failure and delay.

\subsubsection{Greedy Perimeter Stateless Routing with Movement Awareness (GPSR-MA)}

The Greedy Perimeter Stateless Routing with Movement Awareness (GPSR-MA) (Fabrizio, G., Giulia, B., Dzmitry, K., \& Gianni V. 2007) is an enhanced version of the conventional GPSR protocol. GPSR-MA employs a group of routing metrics. Those routing metrics are the velocity (speed and motion direction besides geographical location. The hello Messages (HeM) are transmitted by all participating vehicles in VANET. A HeM packet contains vehicle state (current position, speed, and motion direction). All participating vehicles that receive HeM can build their own neighbours' routing table (NRT). The vehicles that employ GPSR-MA algorithm totally depend only on NRT with its direct neighbours to select its next-hop. Evaluation results showed that GPSR-MA outperformed the original GPSR in terms of packet delivery ratio as well as throughput and reduction in location errors. 


\subsection{Representatives Protocols in Urban Environment}

This section discusses some well-known position-based routing protocols that are deployed in urban environments.

\subsubsection{Greedy Perimeter Stateless Routing with Lifetime (GPSR-L)}

Vehicles that employ the traditional GPSR may select a neighbour that is no longer exists when in reality it remains present in its coverage area. This problem shapes the main issue for routing with GPSR. To deal with such an issue, authors in (Rao, S. A., Pai, M., Boussedjra, M., \&Mouzna, J. 2008, October) proposed the Greedy Perimeter Stateless Routing with Lifetime (GPSR-L). GPSR-L handles the problem based on using the concept of link quality and lifetime. The selection process with the GPSR-L algorithm is accomplished by upgrade the selection of the next-relay vehicle. The forwarding decision is achieved by selecting the one with high link quality and a non-zero lifetime. The proposed approach is calculated using the difference of distances between the nodes computed with the information of two successive Hello Messages. The experiment results showed that GPSR-L outperforms GPSR in terms of packet delivery ratio (PDR) when compared to GPSR.

\subsubsection{A Reliability-Improving Position-Based Routing (RIPR)}

Due to special features of the urban area, the possibility of the void problem and link breaks increases.The author presented in (Ryu, M. W. 2011) a solution to solve those problems. The proposed protocol is the reliability-improving position-based routing (RIPR). The protocol consists of two main modes that are; greedy and recovery mode. All vehicles employ RIPR frequently transmitted beacon messages (BM). A vehicle gets a $\mathrm{BM}$, it estimates the neighbour positions based on velocities and moving directions of vehicles. The greedy mode of RIPR is used for forwarding data packets to the destination through the relay nodes based on the prediction of the new position of neighbours based on the calculations. If greedy mode fails, RIPR switches to the perimeter mode. If the perimeter mode is performed continuously, the packet loss and delay problem will occur because of the extra number of hops used to forward a data packet. To solve this problem, the perimeter mode of RIPR selects the next relay node with a higher density of neighbours, as well as the shortest distance to the destination. The experimental results showed that RIPR outperforms GPSR protocol in terms of packet delivery ratio and the number of link breakage.

\subsubsection{Greedy Routing with Abstract Neighbour Table (GRANT)}

The authors in (Sascha, S., \& Wolfgang, E. 2008) proposed Greedy Routing with Abstract Neighbour Table (GRANT). The GRANT can prevent routed data packets stuck at the void problem. The GRANT is considered one of the prevalent routing protocols that can solve one issue of the conventional GPSR. Vehicles that employ the GRANT maintain a routing table called Abstract Neighbour Table (ANT). The ANT contains the neighbours' state at $\mathrm{x}$-hops. Through using ANT table vehicle can divide the planes into areas and includes a representative neighbour for only one area. Every vehicle applying GRANT algorithm broadcasts its ANT in the beacon message. In GRANT, in order to reduce the extra overhead incurred by beacon messages, the whole plane in ANT is divided into areas and for each area, one representative neighbour is decided.

\section{Performance Evaluations}

The applicability of selected routing protocols in this research is various based on the environment that the routing protocol that will be employed. These variations appeared due to the specification of each protocol, such diversities have a significant impact on the performance of each routing protocol. This paper discusses the properties of the routing protocols and adaptability in both environments. Bellow the specification of simulation parameters for both environments. In this section, the paper evaluates the performance of the selected protocols in NS-2.34. The paper compares the performance of GPSR, GPSR+AGF, GPSR-L, GPSR-MA, GPSR-R, RIPR, GRANT, and PRB-DV in urban and highway environments.

\subsection{Simulation Parameters}

Table 1 below introduces Simulation Parameters for the highway environment and Table 2 shows Simulation Parameters for the urban environment. 
Table 1. Simulation Parameters for highway environment

\begin{tabular}{ll}
\hline Item & Spesefication \\
\hline Simulation & NS2.34 \\
Scenario Area & $5000 \times 5000 \mathrm{~m}$ \\
Simulation time & 300 Seconds \\
Vehicle Nodes & 200 \\
Transmission Range & $250 \mathrm{~m}$ \\
Movement model & Modified Random Waypoint \\
Minimum speed value & $60 \mathrm{~km} / \mathrm{h}$ \\
Maximum speed value & $140 \mathrm{~km} / \mathrm{h}$ \\
Hello packet size & $12 \mathrm{bytes}$ \\
Hello packet interval & $1.5 \mathrm{~second}$ \\
Density between nodes & $5 \mathrm{vehicles}$ every $130 \mathrm{~m}$ \\
MAC layer protocol & IEEE 802.11 DCF \\
Traffic Type & CBR /UDP \\
Packet size & 512 bytes \\
Channel bandwidth & 2 Mbps \\
Radio propagation model & Two Ray Ground Model \\
\hline
\end{tabular}

Table 2. Simulation Parameters for Urban environment

\begin{tabular}{ll}
\hline Item & Spesefication \\
\hline Simulation & NS2.34 \\
Scenario Area & 5000 x $5000 \mathrm{~m}$ \\
Simulation time & 300 Seconds \\
Number of nodes & 200 \\
Transmission Range & $250 \mathrm{~m}$ \\
Movement model & Modified Random Waypoint \\
Minimum speed value & $10 \mathrm{~km} / \mathrm{h}$ \\
Maximum speed value & $50 \mathrm{~km} / \mathrm{h}$ \\
Hello packet size & 12 bytes \\
Hello packet interval & $1.5 \mathrm{~second}$ \\
Density between nodes & $1 \mathrm{vehicles}$ every $10 \mathrm{~m}$ \\
MAC layer protocol & IEEE 802.11 DCF \\
Traffic Type & CBR/UDP \\
Packet size & 512 bytes \\
Channel bandwidth & 2 Mbps \\
Radio propagation model & Two Ray Ground Model \\
\hline
\end{tabular}

\subsection{Simulation Setup}

For urban and highway environments the experiments are carried out in NS-2.34 simulator. This choice is adopted because NS-2.34 simulator is the most simulators used with VANET. Furthermore, this paper deploys the modified random waypoint mobility mode (MRWP) (Sabah, N., \&Hocanin, A. 2010, December). The MRWP model guarantees a moderate speed value relative to the coveted average speed compared to the existing random waypoint (RWP) model. The Packet delivery ratio, void problem occurrence rate (VPOR) and average of hops count in both greedy mode and recovery mode are employed as standards used for the investigations of results. Those metrics are chosen based on the required solution. It was required to be sure that the data is exchanged between the vehicles in different Hops with the highest delivery, and least void occurrence of the surveyed protocols. The performance of the selected metrics has been evaluated in urban and highway environments. The simulation parameters are as shown in table 1 and table 2. Two different scenarios have been conducted for each environment. For the first scenario the source vehicle, intermediate vehicles and destination vehicle travelling in same direction, for the second scenario the source vehicle and destination vehicle travelling in different direction, but the intermediate vehicles are moving in both directions. Further, in each scenario 10 pairs of source-destination are randomly selected. 


\section{Simulation Comparison and Discussion}

\subsection{Highway Environment}

\subsubsection{Scenario1 for Highway Environment}

All participating vehicles are travelling in the same direction. In the first scenario for the highway area, a constant number of 200 vehicles, and a diversity of velocity; 140, 120, 100, 80, and $60 \mathrm{Km} / \mathrm{h}$ were applied. Figure 3 shows the influence of a speed variety on surveyed routing protocols based on the selected performance metrics.

Figure 3(a) shows the packet delivery ratio for GPSR, PBR-DV, GPSR-R, and GPSR-MA. The faster the speed, the fewer packet delivery attained. This is because increasing nodes speed increases topology change rate that increases Link breakage which leads to retransmission which results in increased delay time and finally, packet loss. The packet delivery ratios of PBR-DV is the highest compared to the other protocols.

Figure 3(b) shows the average number of hops for GPSR, PBR-DV, GPSR-R, and GPSR-MA; it can be noticed that whenever the vehicle speed increases; the average number of hops count increases too for all protocols. This is because increasing in nodes speed increases topology change rate that increases Link breakage which leads to increase delay time due to changeover to perimeter mode is a problem caused by transmitting data to the final destination via multiple hops in VANET. GPSR-R and GPSR-MA protocols have slightly the same hops count. PBR-DV has the least hops count compared to the other protocols.

Figure 3(c) shows the void problem occurrence rate for GPSR, PBR-DV, GPSR-R, and GPSR-MA; it can be noticed that the void problem occurrence rate increases as the vehicle speed increases for all protocols. This is because increasing nodes speed increases topology change rate that increases Link breakage which leads to increase changeover to perimeter mode which means more void occurrences in VANET.PBR-DV has the least void problem compared to the other protocols.

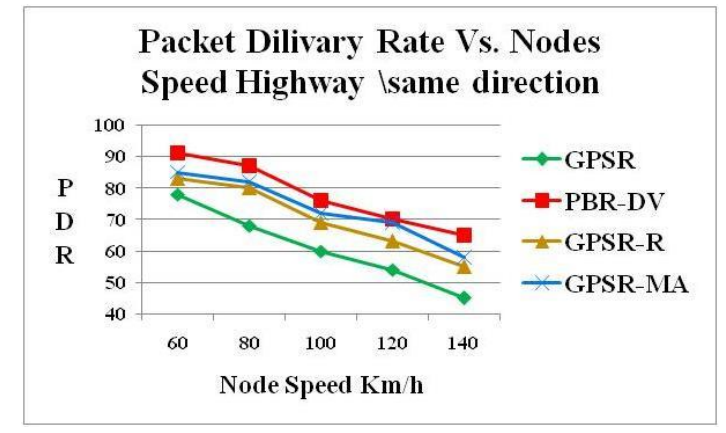

Figure 3a. Shows PDR

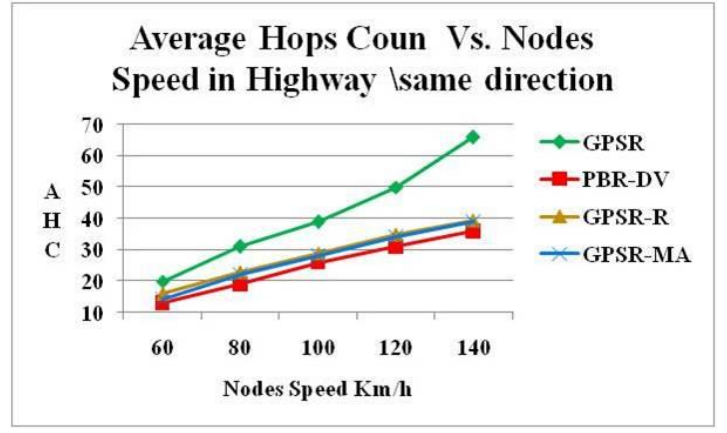

Figure $3 \mathrm{~b}$. shows average hops count

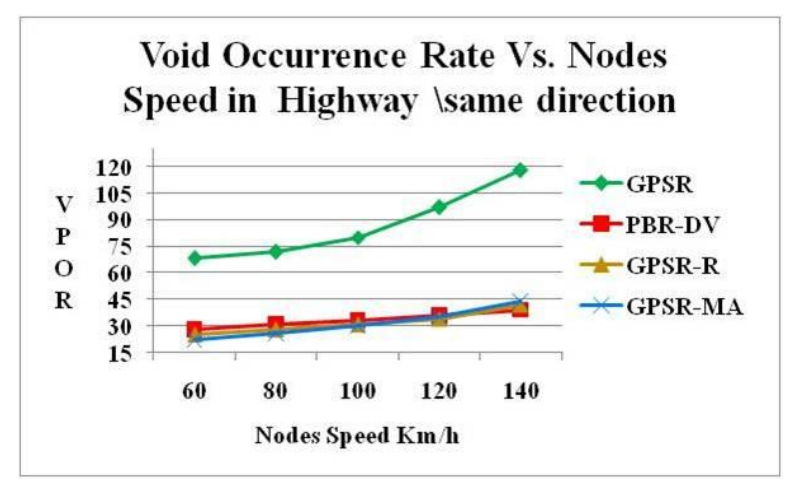

Figure 3c. Shows void problem occurance rate

Figure 3.shows the impact of mobility on surveyed routing protocols based on the selected performance metrics

\subsubsection{Scenario 2 for Highway Environment}

In this scenario, the two communicating nodes travelling in opposite directions other participating nodes are travelling in both directions.In the second scenario for highway area, a constant number of 200 vehicles, and a diversity of movement velocities; 140, 120, 100, 80, and $60 \mathrm{Km} / \mathrm{h}$ were employed. Figure 4 displays the impact of mobility on surveyed routing protocols based on the selected performance metrics. 
Figure 4(a) shows the packet delivery ratio for GPSR, PBR-DV, GPSR-R, and GPSR-MA; it is clear that the faster the speed, the fewer packet delivery rates achieved. This is because increasing nodes' speed increases the topology change rate that increases Link breakage which leads to more packet loss. PBR-DV and GPSR-MA slightly have the same packet delivery ratio; the improvement to GPSR-MA came as a result of its enhanced algorithm in considering the motion direction of nodes.

Figure 4(b) displays the rate of hops count for GPSR, PBR-DV, GPSR-R and GPSR-MA; it can be noticed that the average number of hops decreases as vehicles speed increases for all protocols. This is because scarcity of nodes that share in forwarding process due to link-breakage provides opportunity to reach destination with less hops. PBR-DV and GPSR-MA protocols have slightly the least hops counts compared to other protocols.

Figure 4(c) shows the void problem occurrence rate for GPSR, PBR-DV, GPSR-R and GPSR-MA; it can be noticed that the void problem occurrence rate increases as the speed of node increases for all protocols. This is because less number of nodes provides opportunity to make incorrect routing decision. PBR-DV and GPSR-MA protocols have slightly the least void problem occurrence rate compared to other protocols.

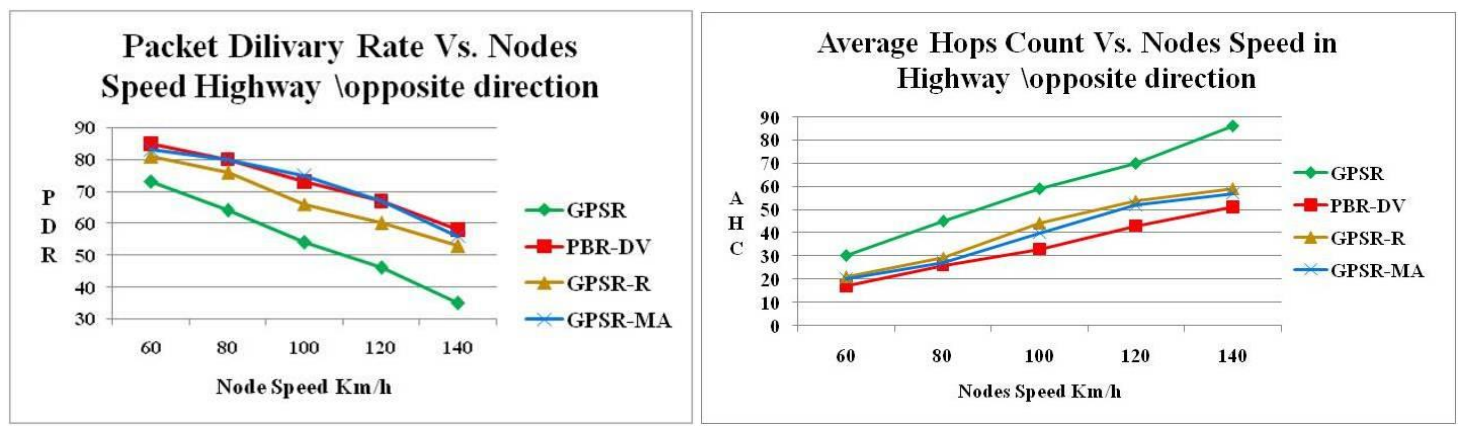

Figure 4a. Shows PDR

Figure $4 \mathrm{~b}$. shows average hops count

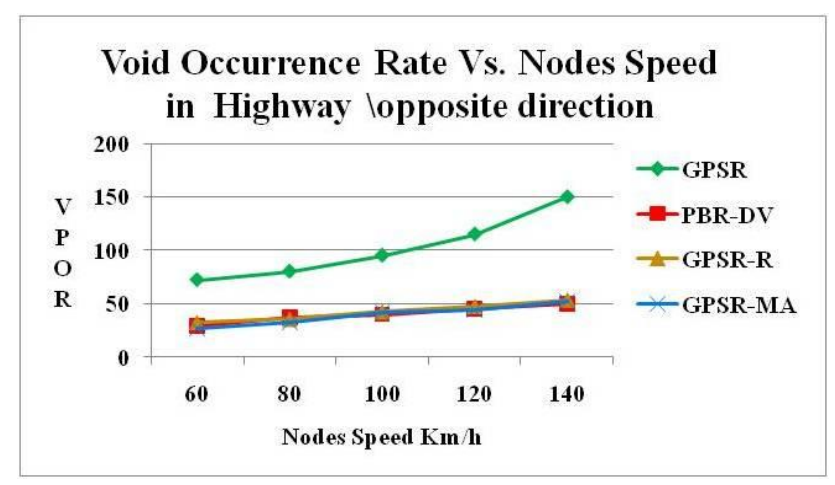

Figure 4c. Shows void problem occurance rate

Figure 4.shows the impact of mobility on surveyed routing protocols based on the selected performance metrics

\subsection{Urban Environment}

\subsubsection{Scenario1 forUrban Environment}

The participating vehicles in VANET are traveling in the same direction. In the first scenario for an urban area, a constant number of 200 vehicles, and a diversity of velocities; 50, 40, 30, 20, and $10 \mathrm{Km} / \mathrm{h}$ were deployed. Figure 5 shows the impact of mobility on surveyed routing protocols based on the selected performance metrics.

Figure 5(a) shows the packet delivery ratio for GPSR, GPSR-L, GRANT, and RIPR; it is clear that the faster the speed, the fewer packet delivery rates is achieved for all protocols. This is because increasing nodes' speed increases the topology change rate that increases Link breakage which leads to packet loss. In this situation, GPSR experiences an increment in packet loss rate, and the packet delivery ratios of GPSR-L and RIPR protocols have the highest slightly the same packet delivery rate compared to GPSR protocol because both make a correct decision in selecting the next hop.

Figure 5(b) shows the hops count ratio for GPSR, GPSR-L, GRANT, and RIPR; it can be noticed that the faster the speed, the fewer hops count ratio is achieved for all protocols. This is due to the fast change in the node's 
neighbours' density which provides an opportunity to reach the destination with fewer hop counts. GPSR-L and RIPR protocols have slightly the same hops count which is less than other protocols.

Figure 5(c) shows the void problem occurrence rate for GPSR, GPSR-L, GRANT and RIPR; it can be noticed that the void problem occurrence rate increases as the speed of nodes increases for all protocols. This is because increasing in nodes speed increases topology change rate that increases Link breakage which leads to face void problem more. GPSR-L and RIPR protocols have slightly the same void problem occurrence rate which is less than other protocols.

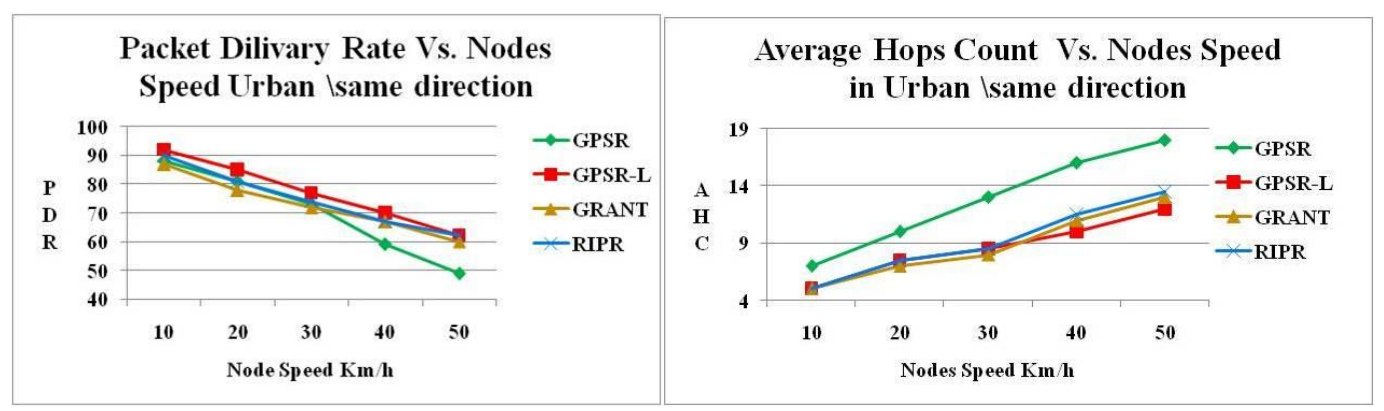

Figure 5a. Shows PDR

Figure5b. shows average hops count

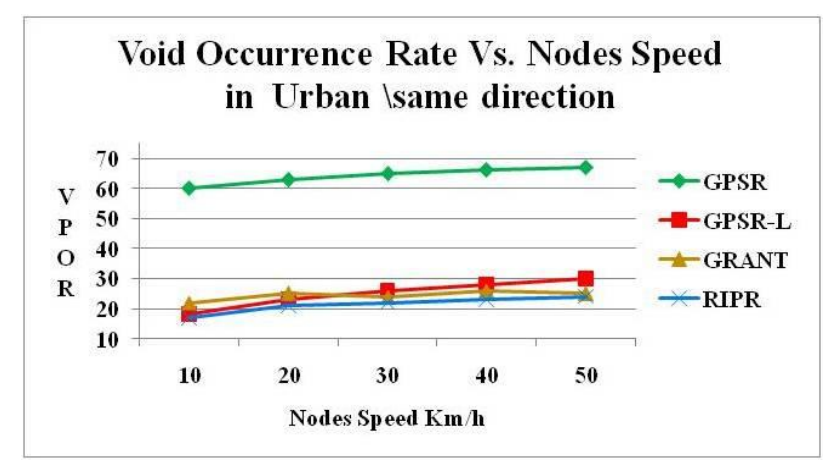

Figure 5c. Shows void problem occurance rate

Figure 5.shows the impact of mobility on surveyed routing protocols based on the selected performance metrics

\subsubsection{Scenario 2 for Urban Environment}

In this scenario, the two communicating vehicles (source and destination) travelling in opposite directions other participating nodes are travelling in both directions. In this scenario for urban areas, a constant number of 200 vehicles, and a diversity of movement speeds; 50, 40, 30, 20, and $10 \mathrm{Km} / \mathrm{h}$ were deployed. Figure 8 shows the impact of mobility on surveyed routing protocols based on the selected performance metrics.

Figure 6(a) shows the packet delivery ratio for GPSR, GPSR-L, GRANT, and RIPR; it can be noticed that the faster the speed, the fewer packet delivery rate achieved by all protocols. The packet delivery ratios of GPSR-L and RIPR protocols have the highest slightly the same packet delivery rate compared to GPSR and GRANT protocols. The improvement on the GPSR-L is referred to its algorithm characteristics.

Figure 6(b) shows the hops count rate for GPSR, GPSR-L, GRANT and RIPR; it can be noticed that the hops count rates decreases as the speed of vehicles increases for all protocols. GPSR-L and RIPR protocols have slightly the least same hops counts compared to other protocols. Also, they deployed motion factor to make routing decision.

Figure 6(c) shows the void problem occurrence rate for GPSR, GPSR-L, GRANT and RIPR; it can be noticed that the void problem occurrence rate increases as the speed of vehicles increases for all protocols. GPSR-L and RIPR protocols have the least void problem compared to the other protocols. This is because they use recovery mode less than other protocols due to their algorithm improvement. Also, they deployed motion factor to make routing decision. 


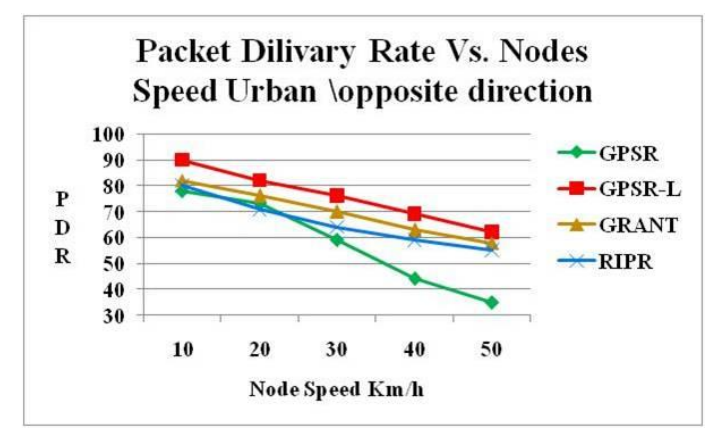

Figure 6a. Shows PDR

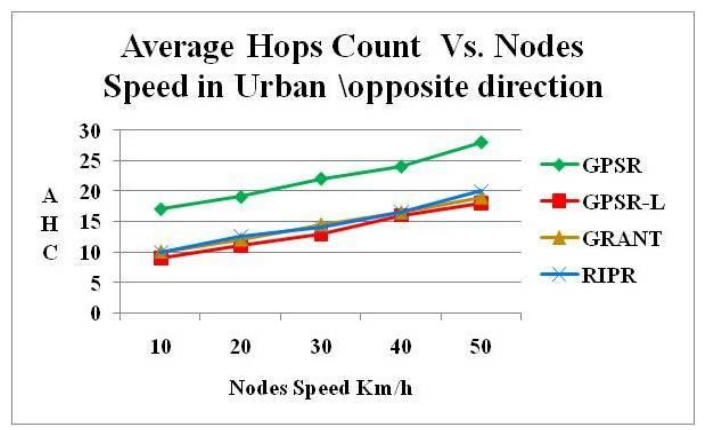

Figure $6 \mathrm{~b}$. shows average hops count

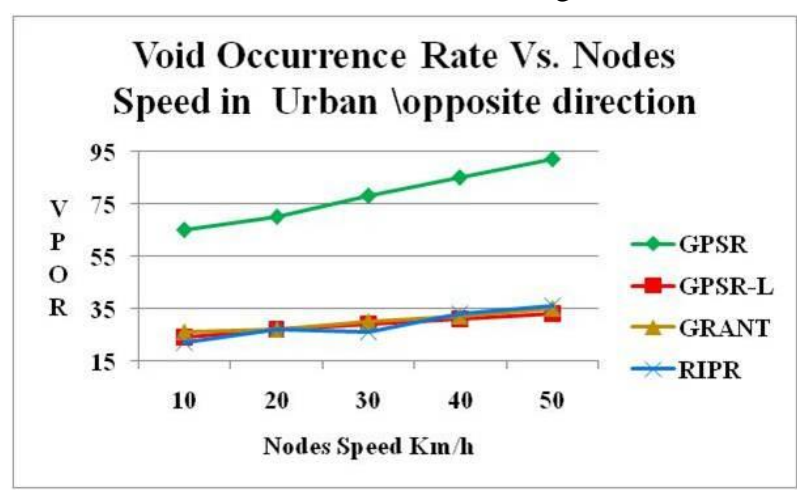

Figure 6c. Shows void problem occurance rate

Figure 6.shows the impact of mobility on surveyed routing protocols based on the selected performance metrics

\section{Conclusion and Recommendations for Future Applicable Routing Protocol}

This paper shed light on several most significant none overly non-DTN position-based routing protocols in urban and highway environments. This paper introduced an intensive evaluation study achieving a performance analysis and comparison of several non-delay tolerant non-overlay routing protocols. Hops count, void problem occurrence rate, and Packet Delivery Ratio were considered as performance metrics. With the simulating VANET operational environment, two experimental settings were taken into account; the effects of diversity of participating vehicles number, and the diversity of vehicles' velocities. Evaluation cropped results in simulation environments have assured the efficiency of non-delay tolerant non-overlay position-based routing protocols in dynamic and dense environments which agrees with qualitative evaluation previously presented by (Singh, P. 2014, April), (Manvendra, S. 2015), (Ali, K., Phillips, I., \& Yang, H. 2016, December). However, some deficiencies observed during the evaluation process should be considered by researchers. The future work direction will develop a routing strategy that is applicable to highways and city environments. That protocol will satisfy the major demands of routing in VANETs.

General talk, the following properties should be considered in the design of the intended future routing protocol. Firstly; it should be adaptively interacting with the unpredictable VANET environment conditions. Therefore, the intended protocol can easily employ the neighbours' degree. Secondly; the design of the intended protocol should consider the nodes' location accuracy that is affected by the variation of nodes' mobility rate. Therefore, the design should be adaptable to the high variation of location information in nodes' routing tables (NRT) through adopting a suitable approach to pull out wrong information from the NRT and improve a credible beaconing updates mechanism. Thirdly, the design also should take into account suitable mechanisms to select the next forwarded vehicle to build an optimal path between communicating vehicles considering several routing metrics such as congestion degree, and connectivity degree nodes, and other metrics. And finally, should be e a cross-layer protocol that considers all layers protocols in the design.

\section{References}

Ali, K., Phillips, I., \& Yang, H. (2016, December ). Evaluating VANET routing in urban environments.2016 39th International Conference on Telecommunications and Signal Processing (TSP), 60-63. https://doi.org/10.1109/TSP.2016.7760829 
Amardeep, D., Harmandeep, S., Sanjay, B., \&Sanjeev, S. (2013, June). Analytical Study of AODV, DSR and DSDV Routing Protocols in VANET simulating City scenario using EstiNet Simulator. Second International Conferenceon Advances in Electronics, Electrical and Computer Engineering - EEC 2013, India, 978-981.

Amina, B., Asmae, B., \& Mohamed, E. (2020). Routing Protocols for VANETs: A Taxonomy, Evaluation and Analysis. Advances in Science, Technology and Engineering Systems Journal, 5(1), 77-85. https://doi.org/10.25046/aj050110

Anil, K. T., \& Ali, T. R. (2016). A Survey on Unicast Routing Protocols for VANET.International Journal of Engineering and Computer Science, 5(5), 16555-16565.

Asim, R, Saira, G., Sana, A., \& Amir, Q. (2017). Vehicular Ad Hoc Network (VANET): A Survey, Challenges, and Applications. Vehicular Ad-Hoc Networks for Smart Cities, Springer Singapore, Singapore, 548, 39-51. https://doi.org/10.1007/978-981-10-3503-6_4

Debasis, D., \& Rajiv, M. (2018). Improvised dynamic network connectivity model for Vehicular Ad-Hoc Networks (VANETs). Journalof Network and Computer Applications, 122, 107-114. https://doi.org/10.1016/j.jnca.2018.08.014

Fabrizio, G., Giulia, B., Dzmitry, K., \& Gianni V. (2007). Enhanced GPSR Routing in Multi-Hop Vehicular Communications through Movement Awareness. in IEEE Communications Letters, 11(10), 781-783. https://doi.org/10.1109/LCOMM.2007.070685

Joilson, A. J., Emilio, C., \& Wille, G. (2018). Routing in Vehicular Ad Hoc Networks: Main Characteristics and Tendencies. Journal of ComputerNetworks and Communications. https://doi.org/10.1155/2018/1302123

Karp, B., \& Kung, H. T. (2000, Aug.). GPSR: Greedy Perimeter Stateless Routing for Wireless Networks. Proceedings of the Annual International Conference on Mobile Compuing and Networking, Mobicom, 2000, New York NY United States.https://doi.org/10.1145/345910.345953

Kaur, H., \& Meenakshi, M. (2017, May). Analysis of VANET geographic routing protocols on real city map. 2nd IEEE International Conference on Recent Trends in Electronics, Information \& Communication Technology (RTEICT), Bangalore, 2017, 895-899. https://doi.org/10.1109/RTEICT.2017.8256727

Kirsch, B., \& Effelsberg, W. (2007). Implementation of a Distance-Vector-Based Recovery-Strategy for Position-Based-Routing, Thesis, Department of Mathematics and Computer Science, University of Mannheim.

Leontiadis, I., \& Mascolo, C. (2007, June). GeOpps: Geographical Opportunistic Routing for Vehicular Networks. IEEE International Symposium on a World of Wireless Mobile and Multimedia Networks, 2007 (WoWMoM 2007), Espoo, Finland, 1-6. https://doi.org/10.1109/WOWMOM.2007.4351688

Manvendra, S. (2015) Non-DTN Geographic Unicast Routing Protocol for VANET: State of the art. International Journal of Current Engineering and Technology, 5(5), 3418-3425.

Perkins, C. E., \& Royer, E. M (1999, Feb). Ad-hoc on-demand distance vector routing. Proceedings WMCSA'99.Second IEEE Workshop on Mobile Computing Systems and Applications. 90-100. https://doi.org/10.1109/MCSA.1999.749281

Rao, S. A., Pai, M., Boussedjra, M., \& Mouzna, J. (2008, Oct.). GPSR-l: Greedy perimeter stateless routing with lifetime for VANETs. in Eighth international conference on ITS tele-communications, 299-304. https://doi.org/10.1109/ITST.2008.4740275

Ryu, M. W. (2011). Position-based Routing Algorithm for Improving Reliability of Inter-Vehicle Communication. TIIS, 5(8), 1388-1403. https://doi.org/10.3837/tiis.2011.08.002

Sabah, N., \&Hocanin, A. (2010, Dec.). Animproved random waypoint mobility model for wireless ad-hoc network.in International Conference on Information and Multimedia Technology (ICIMT 2010), Hong Kong, China, 146-150.

Sascha, S., \& Wolfgang, E. (2008). Position-based unicast routing for city scenarios. International Symposium on a World of Wireless, Mobile and Multimedia Networks, Newport Beach, 1-8.

Shelly, S., \& Babu, A. V. (2015). Link reliability-based greedy perimeter stateless routing for vehicular ad hoc networks. International Journal of Vehicular Technology, 2015(2), 1-16.

https://doi.org/10.1155/2015/921414

Singh, P. (2014, April). Comparative Study Between Unicast and Multicast Routing Protocols in Different Data 
Rates Using Vanet. 2014 International Conference on Issues and Challenges in Intelligent Computing Techniques (ICICT), 278-284. https://doi.org/10.1109/ICICICT.2014.6781293

Smiri, S., Boushaba, A., Ben, A. R., \& Zahi, A. (2018, May). Geographic and topology based routing protocols in vehicular ad-hoc networks: Performance evaluation and QoS analysis, International Conference on Intelligent Systems and Computer Vision (ISCV), Fez, Morocco, 2018, 1-8. https://doi.org/10.1109/ISACV.2018.8354070

Zhao, J., \& Cao, G. (2008). VADD: Vehicle-Assisted Data Delivery in Vehicular ad hoc Networks. In IEEE Transactions on Vehicular Technology, 57, 1910-1922. https://doi.org/10.1109/TVT.2007.901869

Zineb, S. H., \& Imane, Z. (2017). Comparative Study of Routing Protocols Performance for Vehicular Ad-hoc Networks. International Journal of Applied Engineering Research, 12(13), 3867-3878.

\section{Copyrights}

Copyright for this article is retained by the author(s), with first publication rights granted to the journal.

This is an open-access article distributed under the terms and conditions of the Creative Commons Attribution license (http://creativecommons.org/licenses/by/4.0/). 